\title{
Large optical nonlinearity of nanoantennas coupled to an epsilon-near-zero material
}

M. Zahirul Alam ${ }^{1}$, Sebastian A. Schulz ${ }^{1,2,3}$, Jeremy Upham ${ }^{1}$, Israel De Leon ${ }^{4, *}$ and Robert W. Boyd ${ }^{1,5}$

${ }^{1}$ Department of Physics, University of Ottawa, 25 Templeton St., Ottawa, ON, K1N 6N5, Canada.

${ }^{2}$ Centre for Advanced Photonics and Process Analysis, Cork Institute of Technology, Cork, Ireland.

${ }^{3}$ Tyndall National Institute, Cork, Ireland.

${ }^{4}$ School of Engineering and Sciences, Tecnológico de Monterrey, Monterrey, NL 64849, Mexico

${ }^{5}$ Institute of Optics and Department of Physics and Astronomy, University of Rochester, Rochester NY, 14627, USA

*ideleon@itesm.mx

December 13, 2017

The size and operating energy of a nonlinear optical device are fundamentally constrained by the weakness of the nonlinear optical response of common materials. Here, we report that a 50-nm-thick optical metasurface made of optical dipole antennas coupled to an epsilon-near-zero material exhibits a broadband ( $400 \mathrm{~nm}$ bandwidth) and ultrafast (recovery time less than 1 ps) intensitydependent refractive index $n_{2}$ as large as $-3.73 \pm 0.56 \mathrm{~cm}^{2} / \mathrm{GW}$. Furthermore, the metasurface exhibits a maximum optically induced refractive index change of \pm 2.5 over a spectral range of $\sim 200 \mathrm{~nm}$. The inclusion of lowQ nanoantennas on an epsilon-near-zero thin film not only allows one to design a metasur- face with an unprecedentedly large nonlinear optical response but also offers the flexibility to tailor the sign of the response. Our technique allows one to overcome a longstanding challenge in nonlinear optics, namely that of finding a material for which the nonlinear contribution to the refractive index is of the order of unity. It consequently offers the possibility of designing low-power nonlinear nano-optical devices with orders-of-magnitude smaller footprints.

All-optical signal processing and computation are often hailed as breakthrough technologies for the next generation of computation and communication devices. Two important parameters of such devices, energy consumption and size, critically depend on 
the strength of the nonlinear optical response of the materials from which they are made. However, materials typically exhibit an extremely weak nonlinear optical response. This property makes designing subwavelength all-optical active devices extremely difficult. Thus, all-optical active devices tend to have large footprints, which limits the integration density to many orders of magnitude smaller than what can be achieved in a state-of-the-art electronic integrated circuits $[1,2]$. Thus, materials with much stronger nonlinear optical responses are needed in order to enable integrated high-density on-chip nonlinear optical devices.

Over the years several approaches have been explored to enhance the intrinsic nonlinear optical response of materials, including local field enhancement using composite structures [3, 4, 5], plasmonic structures [6, 7], and metamaterials $[8,9,10,11]$. However, these techniques offer only limited control over the magnitude (and sign when applicable) of the wavelength-dependent nonlinear response, and typically involve a trade-off between the strength of the nonlinearity and the spectral position of the peak nonlinear response. It has been reported recently that materials with vanishingly small permittivity commonly known as epsilon-near-zero or ENZ material - exhibit intriguing linear $[12,13,14,15,16]$ and large nonlinear responses $[17,18,19,20,21]$. However, an ENZ material has a large nonlinear response over only a relatively narrow spectral range. Furthermore, the zero-permittivity wavelength, strength of the nonlinear response, and the losses depend on the optical properties of the ENZ material. In comparison to previous works $[17,18]$ where strong nonlinear responses were reported in ENZ materials, here we show that many of these constraints can be overcome by incorporating engineered nanostructures on an ENZ host material. Specifically, we report a new approach to engineer an optical medium with an unprecedentedly large intensity-dependent refractive index using nanoantennas coupled to a thin ENZ material. The simple design concepts presented here provide an exquisite control to engineer the sign and the magnitude of the nonlinear refractive index, and can be used in an all-dielectric CMOS-compatible fabrication process to miniaturize non-linear optical devices by orders of magnitude. We experimentally demonstrate that a metasurface geometry is uniquely suited to engineer a broadband and ultrafast nonlinear response orders of magnitude larger than those of any previously reported solid state materials at optical frequencies.

The rational for using a coupled ENZ-nanoantenna system as the building block for nonlinear optical devices is straightforward. Let us consider an optical antenna placed on a material exhibiting an intensitydependent refractive index. A laser beam that is nearly resonant with such a system experiences a large effective linear refractive index. However, the resonance wavelength of a nanoantenna depends on its geometrical parameters and the refractive index of the surrounding medium. Thus, a small change in the refractive index of the substrate material leads to a spectral shift of the system's linear resonance as a consequence of the dynamic variation of the resonance wavelength of the nanoantenna. As a result, a high-intensity beam at a nearly-resonant wavelength experiences a significantly different refractive index than that experienced by a low-intensity beam, as illustrated in Fig. 1a, resulting in a large effective nonlinear refractive index change of the ENZnanoantenna system, $\Delta n$. Moreover, the field enhancement provided by the nanoantennas decreases the energy requirements for the nonlinear response. As a result, such a system can exhibit a broadband and large intensity-dependent refractive index.

We note that $\Delta n$ can be either positive or negative, depending on the spectral position of the optical signal relative to that of the resonance at a low intensity. Therefore, the sign, magnitude, wavelength position, and bandwidth of $\Delta n$ are no longer constrained by the ENZ substrate, but instead can be tailored by plasmonic or dielectric nanoresonators. These features make the system flexible enough to engineer both the magnitude and sign of the nonlinear response simply by engineering the linear dispersion, by appropriately choosing the antenna parameters,and by choosing an ENZ host that exhibits the required nonlinear response. [17, 18].

We demonstrate the principle of operation using a metasurface $[22,23,24,25,26,27,28]$ consisting of a two-dimensional array of gold optical antennas 
on a thin ENZ layer, which in turn is deposited on a glass substrate (Fig. 1b). In this structure an antenna plays three crucial roles: $(i)$ it dictates the linear dispersion of the overall system, being responsible for a large effective refractive index at frequencies near-resonance; ( $i i)$ it efficiently couples light at normal incidence to the ENZ layer; and (iii) provides a moderate optical field enhancement. We find that the interaction of the antennas with the ENZ layer simulatenously increases the refractive index change $\Delta n$ for a constant incident intensity compared to the bare ENZ, and lowers the intensity threshold for the onset of nonlinear response due to the moderate field enhancement inside the ENZ layer.

We used a 23-nm-thick film of indium tin oxide (ITO) as the ENZ layer. The real part of the permittivity of ITO crosses zero at $\lambda \approx 1420 \mathrm{~nm}$. We chose the geometric parameters of the antenna such that its fundamental dipole resonance occurs at this wavelength. The thin ITO layer supports a lossy bulk-plasma mode at the zero-permittivity wavelength $[29,30,31]$. The linear optical transmission measurement of the metasurface (Fig. 1d) shows two distinct dips which results from the strong coupling-induced splitting between the fundamental mode of the antenna and the bulk-plasma mode of the ENZ layer. The coupled system exhibits two resonances, a main resonance centered at $\lambda=1280 \mathrm{~nm}$, and a weaker resonance centered at $\lambda \approx 1640 \mathrm{nmn}$. The wavelength separation between the resonances ( $330 \mathrm{~nm})$ is larger than the $3 \mathrm{~dB}$ linewidth of the antenna resonance on glass substrate alone $(\sim$ $30 \mathrm{~nm})[32,31]$. This strong coupling plays a significant role in the nonlinear response of the system.

We performed a series of Z-scan measurements [33] for various laser wavelengths to characterize the nonlinear response of the metasurface at normal incidence. We kept the incident intensity at the focus nearly constant at $I \approx 0.150 \mathrm{GW} / \mathrm{cm}^{2}$ for all wavelengths. The polarization of the incident optical field was always along the long axis of the antenna. The metasurface exhibits both an intensity-dependent refractive index and an intensity-dependent absorption. The former is characterized by the effective nonlinear refractive index, $n_{2}=\Delta n / I$, and the latter by the effective nonlinear absorption coefficient, $\beta=\Delta \alpha / I$, where $\Delta n$ and $\Delta \alpha$ are, respectively, the changes in the effective refractive index and in the effective absorption resulting from illuminating the medium with an incident intensity of $I$. The results are shown in Figs. $2 \mathrm{a}$ and $2 \mathrm{~b}$. We find that the nonlinear response is strongly enhanced across the entire spectral range of interest, $1180 \mathrm{~nm} \leq \lambda \leq 1560 \mathrm{~nm}$. The sign and the magnitude of the nonlinear parameters depend on which side of the linear resonance is under investigation, and can be reproduced using a semi-quantitative numerical model describing the red-shift of the linear dispersion of the coupled structure caused by the positive-valued intensity-dependent refractive index of ITO (supplementary information).

The metasurface displays a feature-rich and wavelength-dependent nonlinear response that can be used to control both the magnitude and the sign of the nonlinear parameters. The measured $n_{2}$ (Fig. 2a) show extremely large values at the negative $(\lambda=$ $1240 \mathrm{~nm}$ ) and the positive peaks $(\lambda=1300 \mathrm{~nm})$, which are associated with the main resonance of the system. The absolute maximum response $\left|n_{2}\right|=$ $3.73 \pm 0.56 \mathrm{~cm}^{2} / \mathrm{GW}$ is almost 2000 times larger than the maximum $n_{2}$ of the bare 23-nm-thick ITO at $\lambda=$ $1400 \mathrm{~nm}$ (supplemenatry information) and is three orders of magnitude larger than that of a recently reported highly nonlinear metamaterial [19]. The magnitude of $n_{2}$ steadily decreases as a function of increasing wavelength $(\lambda>1300 \mathrm{~nm})$. Nevertheless, even at a red-detuned wavelength $(\lambda=1480 \mathrm{~nm})$, the nonlinear index, $n_{2}=0.589 \pm 0.088 \mathrm{~cm}^{2} / \mathrm{GW}$, is six orders of magnitude larger than that of silica glass and over two orders of magnitude larger than the nonlinearity of a high-conductivity ITO film at the ENZ region at normal incidence [17]. Similarly, the measured values of $\beta$ (Fig. $2 \mathrm{~b}$ ) have a positive or negative sign depending on the optical wavelength. The values of $\beta$ for $\lambda<1250 \mathrm{~nm}$ and $\lambda>1440 \mathrm{~nm}$ are negative, indicating saturable absorption, whereas the values $1250 \mathrm{~nm}<\lambda<1440 \mathrm{~nm}$ are positive, indicating reverse saturable absorption. The absolute maximum value of $\beta$ is a factor of three smaller than that of a highly nonlinear metamaterial made of structured plasmonic metamolecules [11]. Alternatively, one could design the antenna array in a way to exhibit a much larger $\beta$ range [34] to realize an ultralow- 
power saturable or reverse saturable absorber for applications in ultrafast lasing, optical limiter, and onoff all-optical switching.

The nonlinear dynamics of the overall system is primarily dictated by the nonlinear response of the ITO film [17]. A positive change in the refractive index of ITO - resulting from an ultrafast red-shift of the plasma frequency - leads to the red-shift of the main resonance of the coupled system due to the change in the coupling conditions between the antenna and the bulk plasmon mode of the ITO. Thus a laser pulse at a red-detuned (blue-detuned) wavelength experiences an increase (a decrease) in effective refractive index i.e. a positive (negative) value of the intensity-dependent refractive index. Thus, by choosing appropriate geometric parameters of the antenna array and the zero-permittivity wavelength of the ENZ film, one can control the sign of the nonlinearity.

We studied the temporal dynamics of the resonant nonlinear response using a degenerate pump-probe transmittance measurement at $\lambda=1280 \mathrm{~nm}$. The measurement (Fig. 2c) reveals a rise time of $\sim 260 \mathrm{fs}$, and recovery time of $\sim 600 \mathrm{fs}$. The rise-time measurement was constrained by the temporal width of the pump pulse, suggesting an onset of nonlinearity with sub-100-fs dynamics. The total response time (the rise time plus the recovery time) at the resonant wavelength is only $53 \%$ larger than that of the bare ITO film (supplementary information). The relatively slower response time can be attributed to the resonance effect of the coupled structure. The nonlinear response of the coupled structure can be further improved by using nanoantennas with higher quality factor but possibly at the expense of slower response time [35].

Finally, we investigated the nonlinear contribution to the refractive index at higher intensities by measuring the nonlinear phase shift $\Delta \phi$ as a function of the intensity at four different wavelengths as shown in Fig. 3, two of those tuned to the main resonance $(\lambda=1250 \mathrm{~nm}$ and $\lambda=1265 \mathrm{~nm})$ and two reddetuned from the main resonance $(\lambda=1360 \mathrm{~nm}$ and $\lambda=1440 \mathrm{~nm})$. The maximum resonant nonlinear phase-shift $\Delta \phi=0.677 \mathrm{rad}$ which can occur due to a refractive index change of $\sim-2.74$ for propagation through a 50-nm-thick material. Even at $\lambda=1440 \mathrm{~nm}$ (a highly red-detuned wavelength) the change to the refractive index is $\sim 2.6$ while the change in the transmittance is less than $6 \%$. Such a broadband, large, and ultrafast optically induced change in the refractive index is unprecedented. For most cases, saturation effects become evident for incident energy density larger than $\sim 2 \mathrm{pJ} / \mu \mathrm{m}^{2}$, which is lower than the damage threshold of the gold antennas. The saturation of $\Delta n$ can be attributed to various causes including the large damping of the low conductivity ITO, which limits the maximum achievable temperature of the free electrons. Furthermore, the increased electron temperature spectrally widens the resonance of the coupled structure limiting the maximum phase shift achievable. Thus, ENZ material with lower intrinsic damping could increase the saturation threshold. The nonlinear response could be further enhanced by maximizing the overlap between the near-field of the antennas and the ITO layer, by choosing antennas (dielectric or metallic) with higher quality factors, and by choosing an ENZ material with lower intrinsic damping $[18,36]$.

We note that when the ENZ medium is sufficiently thick, the antenna resonance gets locked to the zero permittivity wavelength of the ENZ material [37]. Nevertheless a metasurface that incorporates a thick ENZ material will also exhibit enhanced nonlinear response because a dynamic change in the substrate's refractive index necessarily modifies the resonance wavelength of the antenna. The strategy introduced in this paper can be considered as a temporal analogue of the typical space-gradient metasurface $[22,23,24,25,27]$. Thus, by combining both strategies one could design an all-optical space-time gradient metasurface to break the geometrical and temporal symmetry on-chip [38, 39], design ultrafast temporal holograms, and control the directivity of emission using an optical gate signal [40, 41].

In summary, we have demonstrated that a metasurface geometry is uniquely suited to achieving large nonlinear refraction. We have shown that a metasurface may exhibit an extremely large intensitydependent refractive index if an epsilon-near-zero medium is incorporated into the design. The technique introduced in this paper offers flexibility in cus- 
tomizing both the wavelength-dependent magnitude and the signs of the nonlinear refractive index and the nonlinear absorption coefficient in any optical frequency of interest. The 50-nm-thick proof-of-concept metasurface exhibits a large broadband nonlinear response with sub-picosecond recovery time. The maximum absolute value of the nonlinear refractive index of the metasurface is three orders of magnitude larger than the bare, planar ITO at normal incidence [17]. As a benchmark comparison, it is also seven orders of magnitude larger than that of glass, four orders of magnitude larger than those of GaAs and $\mathrm{As}_{2} \mathrm{~S}_{3}$ [42]. However, the large absorption in the coupled structure is a constraint. From the operational point of view, perhaps a more important criterion is the maximum achievable nonlinear phase shift over one absorption length. The maximum theoretical absorption in the structure, including the ohmic loss in metal, over the entire spectral range of interest is less than 40\% (supplementary information). Further improvements to the design and material selection, such as dielectric nanoantennas and an ENZ material with less damping, may reduce the overall absorption while increasing the efficiency. More interestingly, the magnitude of the maximum nonlinear contribution to the refractive index $(>2.5)$ is comparable to or larger than the linear refractive index of typical optical materials. Our findings introduce a new paradigm for developing efficient nonlinear-medium for applications involving low-power, high-integration-density nonlinear nanophotonic devices, and also demonstrate a subwavelength nonlinear metasurface for ultrafast all-optical control of the phase, amplitude and the polarization of light.

\section{Methods}

Design: We used a comercial FDTD software (Lumerical FDTD solutions) to design the metasurface. The material dispersion was taken into account by using experimental values for the permittivity of ITO. The permittivity of the ITO film was measured using a spectroscopic ellipsometer. The antenna was designed by choosing the length-to-width ratio in such a way so that the fundamental dipole resonance of the antenna in the absence of the ITO film (i.e. assuming only the glass substrate) lies near the zero crossing wavelength of the permittivity of the ITO film. The fabricated antenna's have an average dimension of $\sim 370 \mathrm{~nm} \times 110 \mathrm{~nm} \times 27 \mathrm{~nm}$ (designed dimension is $\sim 355 \mathrm{~nm} \times 110 \mathrm{~nm} \times 27 \mathrm{~nm})$ and the unit cell dimension is $600 \mathrm{~nm} \times 600 \mathrm{~nm}$. The ITO film is $23 \mathrm{~nm}$ thick with surface resistivity of $\sim 80 \Omega / \mathrm{sq}$.

Fabrication: We fabricated a $500 \mu \mathrm{m} \times 500 \mu \mathrm{m}$ antenna array on a commercially available ITO on a glass substrates (PGO GMBH). The antenna patterns were defined in a bilayer PMMA resist using electron beam lithography (Raith Pioneer $30 \mathrm{kV}$ ), followed by a $\mathrm{Au}$ deposition and lift-off step. Intraparticle proximity error correction was used to ensure sharp corners and a good rectangular shape of the antennas [43].

Measurements: We measured the linear response of the metasurface using a thermal light source and a custom-built transmittance measurement system. We used a single-beam Z-scan technique to measure the nonlinear response of the system using a regeneratively amplified femtosecond laser pumped OPA (optical parametric amplifier) as a the source laser. The pulse width was $\sim 140$ fs. We used a pair of apertures to produce a beam with circularly symmetric trimmed Airy profile (that is, the central lobe only of an Airy pattern) in the far-field. A pair of achromatic doublet lenses was then used to collimate and enlarge the beam. Part of the collimated beam was routed to a reference photodiode to monitor power fluctuations. The rest of the collimated beam was then focused onto the sample using an achromatic doublet lens with anti-reflection coating. The diffracted light after the sample was split using a pellicle beam splitter and routed to a pair of detectors for closed- and open-aperture measurements. The sample was mounted on a computer-controlled motorized translation stage. For each position of the sample we record the truncated mean values (the lowest $25 \%$ and the highest $25 \%$ values were discarded before calculating the mean) from the photodiodes averaged over roughly 3000 pulses. Due to the diffractive propagation required to produce a collimated beam with an Airy profile, the beam diameter at the Z-scan lens varied as a function of wavelength. 
Specifically, the input beam diameter varied from $10.2 \pm 0.15 \mathrm{~mm}$ to $14.65 \pm 0.15 \mathrm{~mm}$ for $\lambda=1160 \mathrm{~nm}$ to $\lambda=1560 \mathrm{~nm}$. The beam was focused onto the sample using an anti-reflection coated achromatic doublet lens of $\sim 100 \mathrm{~mm}$ focal length. The closed-aperture detector was placed $285 \mathrm{~mm}$ away from the Z-scan lens. We did not use any aperture before the closedaperture detector because the active area $(5 \mathrm{~mm}$ diameter) of the detector was much smaller than the beam diameter. The use of an even smaller aperture adversely affected the measurement by reducing the signal-to-noise ratio.

We used the Fresnel-Kirchhoff diffraction integral with a fast-Fourier-transform-based beam propagation method to simulate the experimental beam propagation over the entire setup. We were thus able to calculate the z-dependence of the light falling onto the closed- and open-aperture photodiodes. We wrote the numerical beam propagation program using Julia language [44], and it allows arbitrary order nonlinear refraction and absorption to be taken into account. We have observed that at high intensity fifth-order nonlinear absorption becomes significant and at nonresonant wavelengths can almost completely cancel the third-order nonlinear absorption.

Numerical model of the nonlinear refractive index: To calculate the nonlinear refractive index we do a series of linear FDTD simulations of the antenna-ITO structure by varying the plasma frequency of the ITO. Then we calculate the homogenized refractive index from each set of s-parameters resulting in plasma frequency-dependent effective index distribution of the coupled system. We then calculate the time-dependent electronic temperature using a semiclassical two-temperature model. We calculate the plasma frequency of the ITO layer based on the electronic temperature (supplementary information). Based on the calculated plasma frequency and the set of homogenized refractive indices, we calculate the time dependent change in the refractive index. We calculate the effective nonlinear coefficients $n_{2}$ and $\beta$ by dividing the time-averaged homogenized refractive index (normalized by the pulse shape) by the incident intensity. In this model we ignore the intrinsic nonlinearity of the metal and the change in absorption of the coupled system due to the temperature dependent change in the damping coefficient (imaginary part of the permittivity) of the ITO layer. This leads to an overestimation of $\beta$ obtained from the numerical model for all wavelengths far from the saturation intensity. Hence, we scale down $\beta$ values by a factor of 3.0 to fit the numerical result to that obtained in the experiment.

Data Availability: All relevant data and codes used in this paper are available can be obtained from the authors.

\section{Author Contributions}

I.D.L. conceived the idea and initiated the study. I.D.L., S.A.S., M.Z.A., J.U. designed the sample. S.A.S. performed the FDTD simulations. S.A.S. and J.U. fabricated the sample. M.Z.A. performed all experiments and the corresponding data analysis, developed the numerical model to describe the nonlinear response and wrote the first draft. All authors contributed in finalizing the manuscript. I.D.L. and R.W.B. supervised the project.

\section{Acknowledgement}

The authors gratefully acknowledge the support of the Canada Excellence Research Chairs Program. R.W.B. is the co-founder and Chief Technology Officer of KBN Optics, Pittsford NY. I.D.L. acknowledges financial support from CONACyT (Ciencia Básica) grant 286150. M.Z.A. thanks Saumya Choudhary for the discussion on the linewidth broadenning mechanisms in a plasmonic system.

\section{References}

[1] Miller, D. A. B. Are optical transistors the logical next step? Nature Photonics 4, 3-5 (2010).

[2] Sherwood-Droz, N. et al. Optical $4 \times 4$ hitless silicon router for optical networks-on-chip (noc). Optics express 16, 15915-15922 (2008). 
[3] Boyd, R. W., Gehr, R. J., Fischer, G. L. \& Sipe, J. Nonlinear optical properties of nanocomposite materials. Pure and Applied Optics: Journal of the European Optical Society Part A 5, 505 (1996).

[4] Boyd, R. W. \& Sipe, J. E. Nonlinear optical susceptibilities of layered composite materials. JOSA B 11, 297-303 (1994).

[5] Sarychev, A. K. \& Shalaev, V. M. Electromagnetic field fluctuations and optical nonlinearities in metal-dielectric composites. Physics Reports 335, 275-371 (2000).

[6] Abb, M., Albella, P., Aizpurua, J. \& Muskens, O. L. All-optical control of a single plasmonic nanoantenna-ito hybrid. Nano letters 11, 24572463 (2011).

[7] Abb, M., Wang, Y., De Groot, C. \& Muskens, O. L. Hotspot-mediated ultrafast nonlinear control of multifrequency plasmonic nanoantennas. Nature communications 5 (2014).

[8] Lee, J. et al. Giant nonlinear response from plasmonic metasurfaces coupled to intersubband transitions. Nature 511, 65-69 (2014).

[9] Yang, Y. et al. Nonlinear fano-resonant dielectric metasurfaces. Nano letters 15, 7388-7393 (2015).

[10] Minovich, A. E. et al. Functional and nonlinear optical metasurfaces. Laser $\&$ Photonics Reviews 9, 195-213 (2015).

[11] Ren, M. et al. Nanostructured plasmonic medium for terahertz bandwidth all-optical switching. Advanced Materials 23, 5540-5544 (2011).

[12] Liberal, I., Mahmoud, A. M., Li, Y., Edwards, B. \& Engheta, N. Photonic doping of epsilon-near-zero media. Science 355, 10581062 (2017).

[13] Engheta, N. Pursuing near-zero response. Science 340, 286-287 (2013).
[14] Edwards, B., Alù, A., Young, M. E., Silveirinha, M. \& Engheta, N. Experimental verification of epsilon-near-zero metamaterial coupling and energy squeezing using a microwave waveguide. Physical review letters 100, 033903 (2008).

[15] Alù, A., Silveirinha, M. G., Salandrino, A. \& Engheta, N. Epsilon-near-zero metamaterials and electromagnetic sources: Tailoring the radiation phase pattern. Physical Review B 75, 155410 (2007).

[16] Silveirinha, M. \& Engheta, N. Tunneling of electromagnetic energy through subwavelength channels and bends using $\varepsilon$-near-zero materials. Physical review letters 97, 157403 (2006).

[17] Alam, M. Z., De Leon, I. \& Boyd, R. W. Large optical nonlinearity of indium tin oxide in its epsilon-near-zero region. Science 352, 795-797 (2016).

[18] Caspani, L. et al. Enhanced nonlinear refractive index in $\varepsilon$-near-zero materials. Physical Review Letters 116, 233901 (2016).

[19] Neira, A. D. et al. Eliminating material constraints for nonlinearity with plasmonic metamaterials. Nature communications 6 (2015).

[20] Capretti, A., Wang, Y., Engheta, N. \& Dal Negro, L. Enhanced third-harmonic generation in si-compatible epsilon-near-zero indium tin oxide nanolayers. Optics letters 40, 1500-1503 (2015).

[21] Luk, T. S. et al. Enhanced third harmonic generation from the epsilon-near-zero modes of ultrathin films. Applied Physics Letters 106, 151103 (2015).

[22] $\mathrm{Yu}, \mathrm{N}$. et al. Light propagation with phase discontinuities: generalized laws of reflection and refraction. Science 334, 333-337 (2011).

[23] Ni, X., Emani, N. K., Kildishev, A. V., Boltasseva, A. \& Shalaev, V. M. Broadband light bending with plasmonic nanoantennas. Science $\mathbf{3 3 5}$, 427-427 (2012). 
[24] Kildishev, A. V., Boltasseva, A. \& Shalaev, V. M. Planar photonics with metasurfaces. Science 339, 1232009 (2013).

[25] Yu, N. \& Capasso, F. Flat optics with designer metasurfaces. Nature materials 13, 139150 (2014).

[26] High, A. A. et al. Visible-frequency hyperbolic metasurface. Nature 522, 192-196 (2015).

[27] Lin, D., Fan, P., Hasman, E. \& Brongersma, M. L. Dielectric gradient metasurface optical elements. Science 345, 298-302 (2014).

[28] Chong, K. E. et al. Polarization-independent silicon metadevices for efficient optical wavefront control. Nano letters 15, 5369-5374 (2015).

[29] Vassant, S. et al. Epsilon-near-zero mode for active optoelectronic devices. Physical review letters 109, 237401 (2012).

[30] Campione, S., Brener, I. \& Marquier, F. Theory of epsilon-near-zero modes in ultrathin films. Phys. Rev. B 91, 121408 (2015).

[31] Campione, S., Wendt, J. R., Keeler, G. A. \& Luk, T. S. Near-infrared strong coupling between metamaterials and epsilon-near-zero modes in degenerately doped semiconductor nanolayers. ACS Photonics 3, 293-297 (2016).

[32] Schulz, S. A. et al. Optical response of dipole antennas on an epsilon-near-zero substrate. Phys ical Review A 93, 063846 (2016).

[33] Sheik-Bahae, M., Said, A. A., Wei, T.-H., Hagan, D. J. \& Van Stryland, E. W. Sensitive measurement of optical nonlinearities using a single beam. IEEE Journal of Quantum Electronics 26, 760-769 (1990).

[34] Luk, T. S. et al. Directional perfect absorption using deep subwavelength low-permittivity films. Physical Review B 90, 085411 (2014).

[35] Khurgin, J. B., Sun, G., Chen, W. T., Tsai, W.Y. \& Tsai, D. P. Ultrafast thermal nonlinearity. Scientific Reports 5 (2015).
[36] Sachet, E. et al. Dysprosium-doped cadmium oxide as a gateway material for mid-infrared plasmonics. Nature materials 14, 414-420 (2015).

[37] Kim, J. et al. Role of epsilon-near-zero substrates in the optical response of plasmonic antennas. Optica 3, 339-346 (2016).

[38] Hadad, Y., Sounas, D. \& Alu, A. Space-time gradient metasurfaces. Physical Review B $\mathbf{9 2}$ 100304 (2015).

[39] Yu, Z. \& Fan, S. Complete optical isolation created by indirect interband photonic transitions. Nature photonics 3, 91-94 (2009).

[40] Staude, I. et al. Tailoring directional scattering through magnetic and electric resonances in subwavelength silicon nanodisks. ACS nano 7 , 7824-7832 (2013).

[41] Kuznetsov, A. I., Miroshnichenko, A. E., Brongersma, M. L., Kivshar, Y. S. \& Luk'yanchuk, B. Optically resonant dielectric nanostructures. Science 354, aag2472 (2016).

[42] Boyd, R. W. Nonlinear optics (Academic press, 2003)

[43] Schulz, S. A. et al. Quantifying the impact of proximity error correction on plasmonic metasurfaces [invited]. Optical Materials Express 5, 2798-2803 (2015).

[44] Bezanson, J., Edelman, A., Karpinski, S. \& Shah, V. B. Julia: A fresh approach to numerical computing. SIAM Review 59, 65-98 (2017).

[45] Campione, S., Kim, I., de Ceglia, D., Keeler, G. A. \& Luk, T. S. Experimental verification of epsilon-near-zero plasmon polariton modes in degenerately doped semiconductor nanolayers. Optics Express 24, 18782-18789 (2016).

[46] Liu, X. et al. Quantification and impact of nonparabolicity of the conduction band of indium tin oxide on its plasmonic properties. Applied Physics Letters 105, 181117 (2014). 
[47] Kane, E. O. Band structure of indium antimonide. Journal of Physics and Chemistry of Solids 1, 249-261 (1957).

[48] Chakravarti, A. \& Nag, B. Generalized einstein relation for degenerate semiconductors having non-parabolic energy bands. International Journal of Electronics Theoretical and Experimental 37, 281-284 (1974).

[49] Guo, P., Schaller, R. D., Ketterson, J. B. \& Chang, R. P. H. Ultrafast switching of tunable infrared plasmons in indium tin oxide nanorod arrays with large absolute amplitude. Nature Photonics 10, 267-273 (2016).

[50] Guo, P. et al. Large optical nonlinearity of ito nanorods for sub-picosecond all-optical modulation of the full-visible spectrum. Nature Communications 7, 12892 (2016).

[51] Brorson, S., Fujimoto, J. \& Ippen, E. Femtosecond electronic heat-transport dynamics in thin gold films. Physical Review Letters 59, 1962 (1987).

[52] Sun, C.-K., Vallee, F., Acioli, L., Ippen, E. \& Fujimoto, J. Femtosecond investigation of electron thermalization in gold. Physical Review $B$ 48, 12365 (1993).

[53] Carpene, E. Ultrafast laser irradiation of metals: Beyond the two-temperature model. Physical Review B 74, 024301 (2006).

[54] Lin, Z., Zhigilei, L. V. \& Celli, V. Electronphonon coupling and electron heat capacity of metals under conditions of strong electronphonon nonequilibrium. Physical Review B 77, 075133 (2008).

[55] Yagi, T. et al. Analysis on thermal properties of tin doped indium oxide films by picosecond thermoreflectance measurement. Journal of Vacuum Science \& Technology A 23, 1180-1186 (2005).

[56] Voisin, C., Fatti, N. D., Christofilos, D. \& Vallée, F. Ultrafast electron dynamics and optical nonlinearities in metal nanoparticles. The Journal of Physical Chemistry B 105, 2264-2280 (2001).
[57] Smith, D. R., Schultz, S., Markoš, P. \& Soukoulis, C. Determination of effective permittivity and permeability of metamaterials from reflection and transmission coefficients. Physical Review B 65, 195104 (2002). 

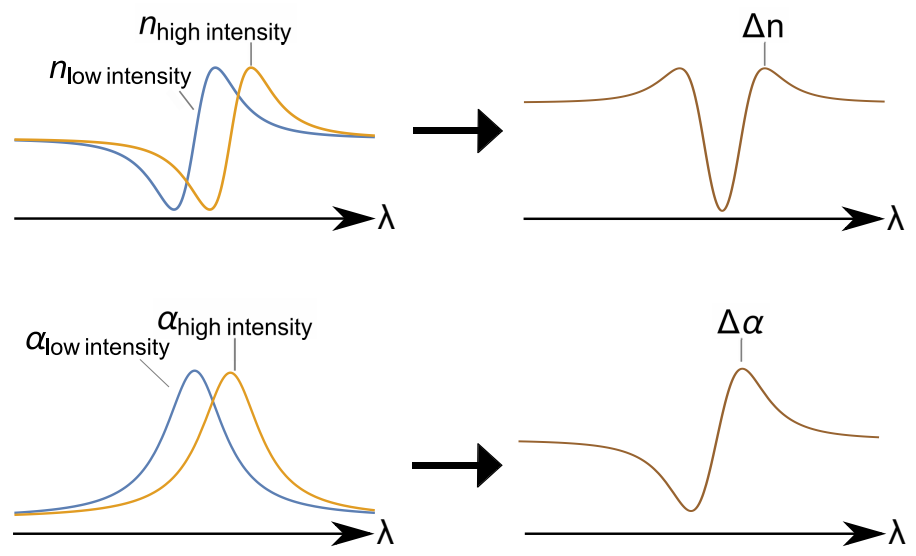

(a)

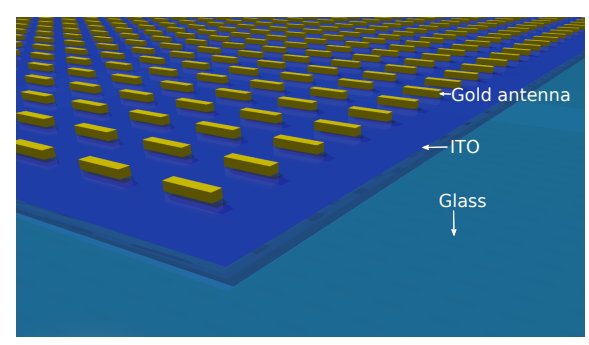

(b)

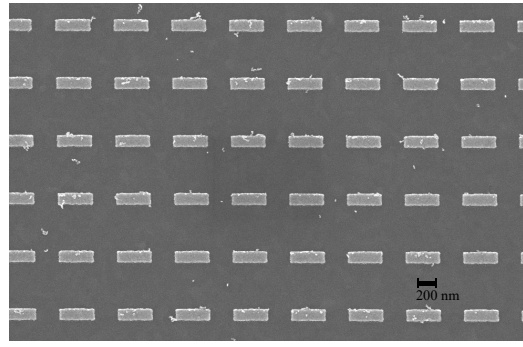

(c)

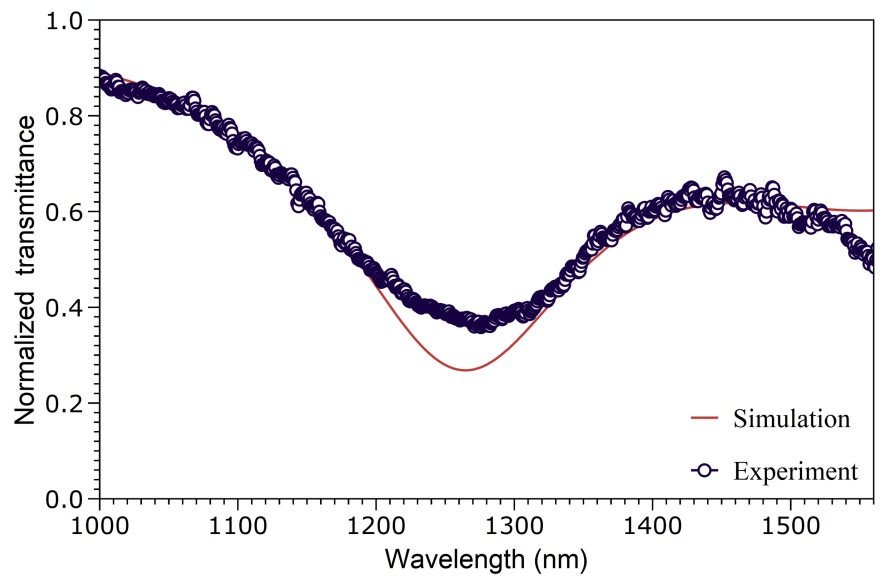

(d)

Figure 1: Design concept and the linear response of the coupled structure. (a) A small nonlinear change of the substrate material's refractive index results in a significant spectral-shift of the resonance wavelength of an antenna. As a result, light of high intensity experiences a dramatically different refractive index $(\Delta n)$ and a different absorption $(\Delta \alpha)$ than those experienced by low intensity light. (b) Our structure is made of a gold dipole antenna array of $27 \mathrm{~nm}$ thickness on a glass substrate. A 23-nm-thick ITO layer is sandwiched between the antenna array and the glass substrate. (c) An SEM micrograph of the fabricated antenna array. (d) The thin ENZ medium supports a bulk plasma mode near the zero permittivity wavelength $[30,45]$. When a dipole antenna array woth resonance at $\sim 1420 \mathrm{~nm}$ (due to the presence of the glass substrate only) is placed on an ITO-glass substrate, the transmission curve shows a splitting of the transmission resonance due to the strong coupling between the fundamental mode of each antenna and the ENZ mode [32, 31]. The variation between the experimental result and the FDTD simulation - with antennas of dimension $362 \mathrm{~nm} \times 108 \mathrm{~nm} \times 27 \mathrm{~nm}-$ can be attributed to inhomogeneous broadening of the linewidth of the antennas due to fabrication imperfections. 


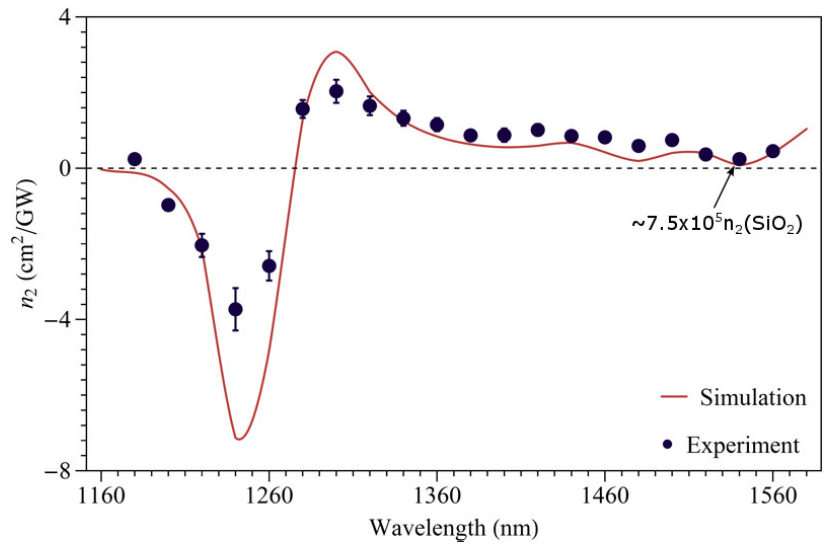

(a)

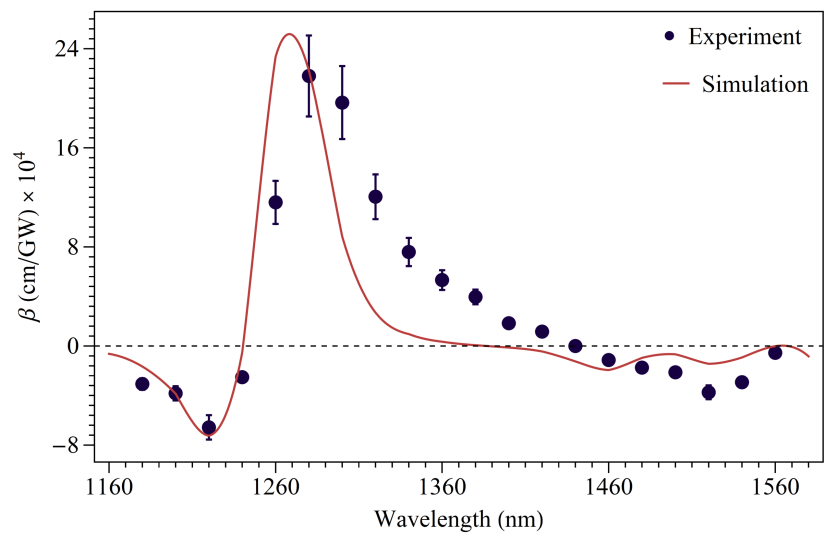

(b)

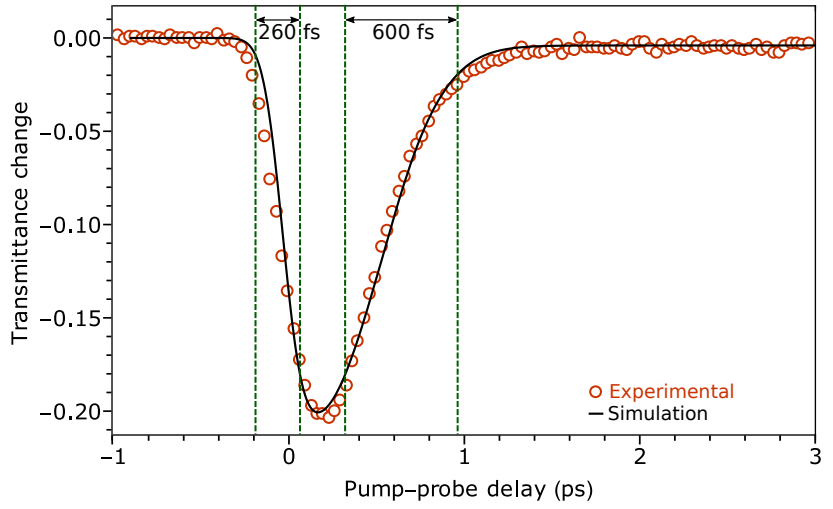

(c)

Figure 2: Nonlinear response of the coupled system. (a) The effective nonlinear refractive index $n_{2}$ as measured using a Z-scan technique. The metasurface exhibits a large nonlinear response. The intensitydependent refractive index of the metasurface is almost six orders of magnitude larger than that of $\mathrm{SiO}_{2}$ even for a highly red-detuned wavelength $(\lambda=1540 \mathrm{~nm})$. (b) The nonlinear absorption $\beta$. The solid lines show the simulated $n_{2}$ and $\beta$ values as calculated using a two-temperature model while taking the non-parabolic conduction band structure of the ITO $\mathrm{I}^{1}$ nto consideration (supplementary information). The semi-quantitative numerical simulation predicts the main features of the wavelength-dependent nonlinear response. The error bars correspond to the uncertainity in measured values due to distortion of the z-scan traces. (c) The pump-induced change in the transmittance of the probe at $1280 \mathrm{~nm}$. The dashed vertical lines represent the 10\% - 90\% transient time. The slow recovery time is dictated by the electron-phonon coupling strength and the quality factor of the resonance of the coupled system. 

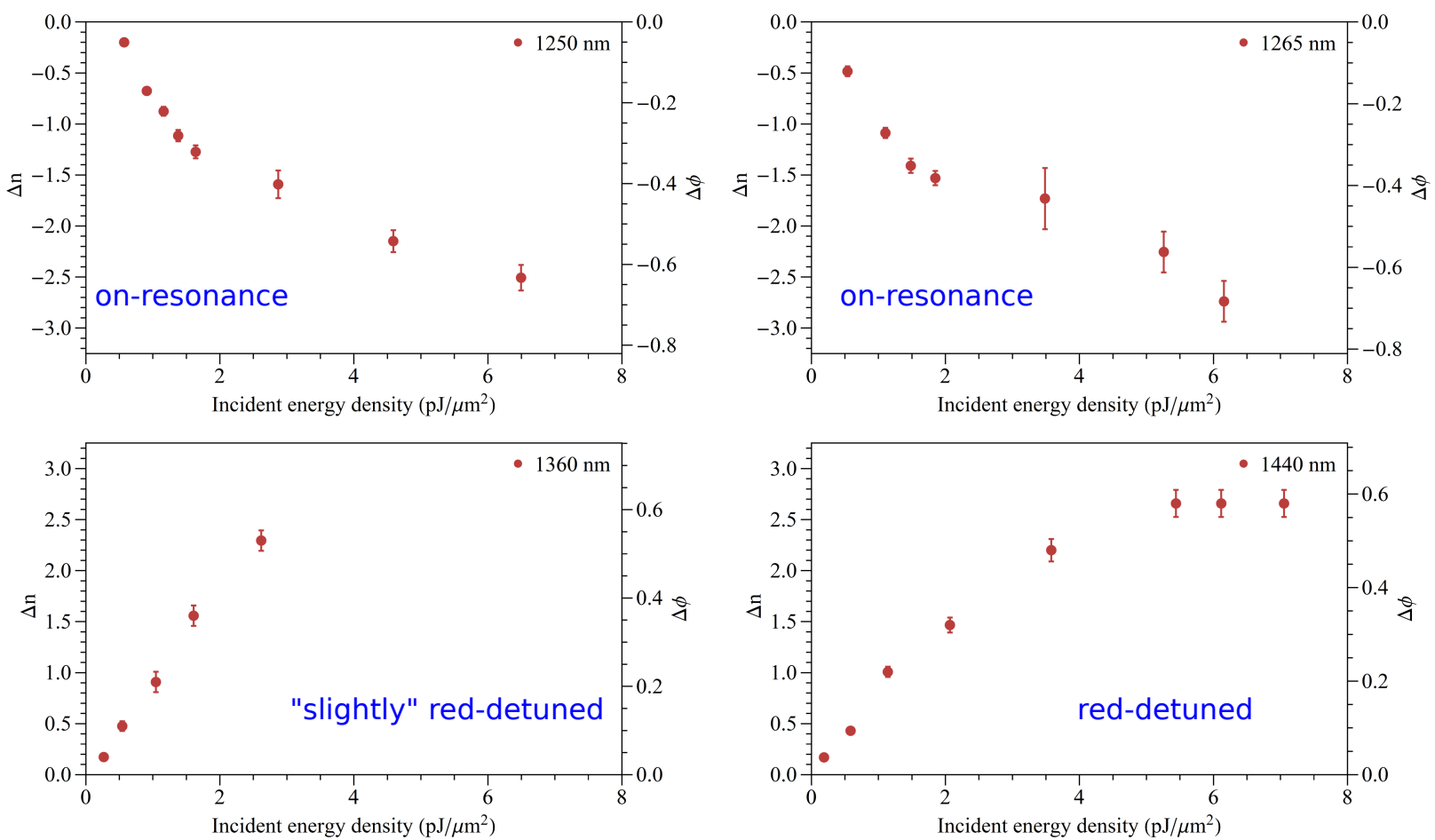

Figure 3: Maximum nonlinear contribution to the refractive index as a function of incident energy density. We determined the maximum nonlinear contribution to the refractive index by measuring the phase shifts at four different wavelengths for a series of different incident energy densities. Even at a highly red-detuned wavelength of $1440 \mathrm{~nm}$ the maximum nonlinear contribution to the refractive index is larger than 2.5. These results show that the metasurface exhibits a large refractive index change across a wide bandwidth. We note that the saturation effects present in the plots can be modelled to extract effective fifth-order nonlinear coefficients. 
(a)
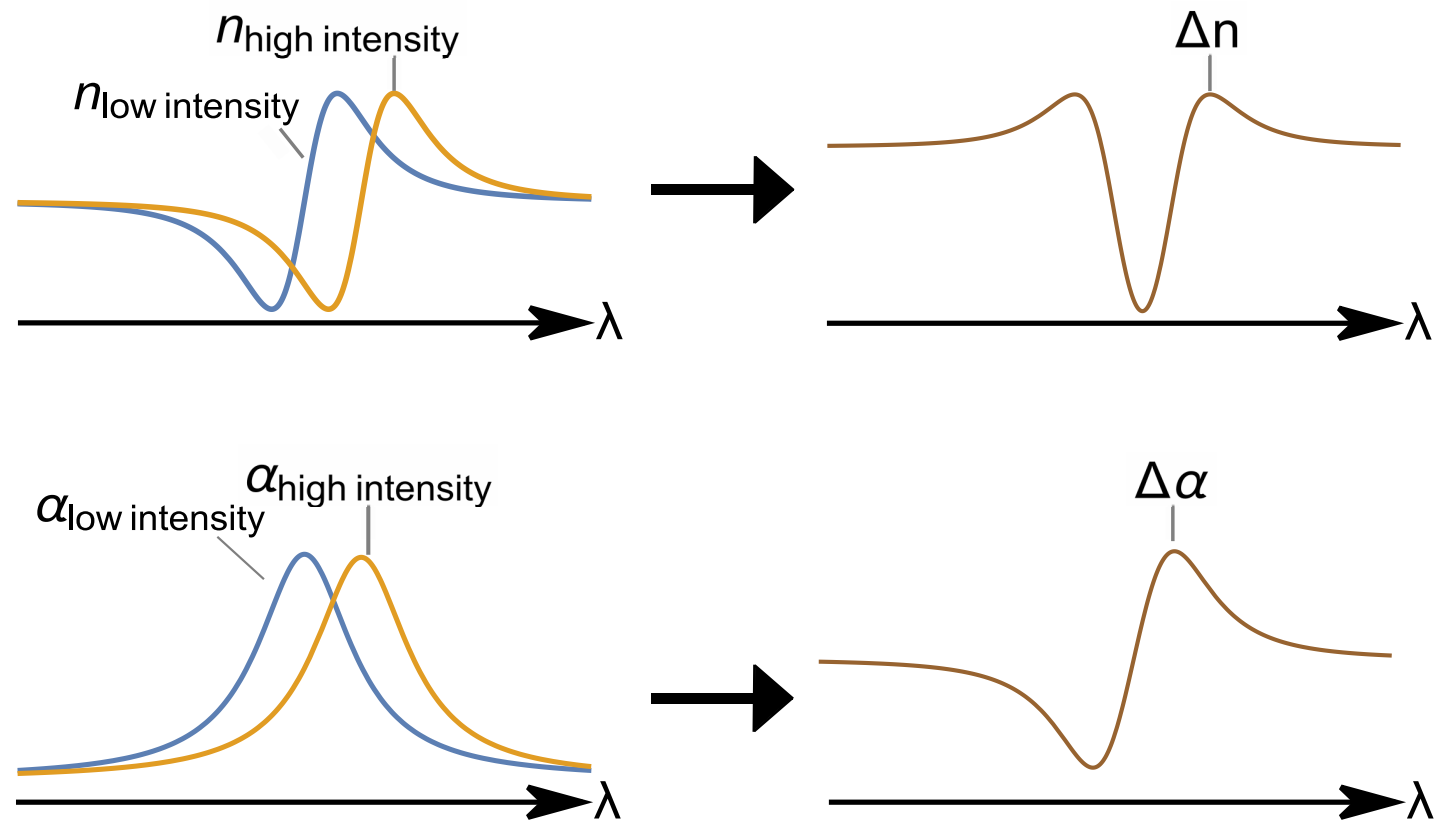

(b)

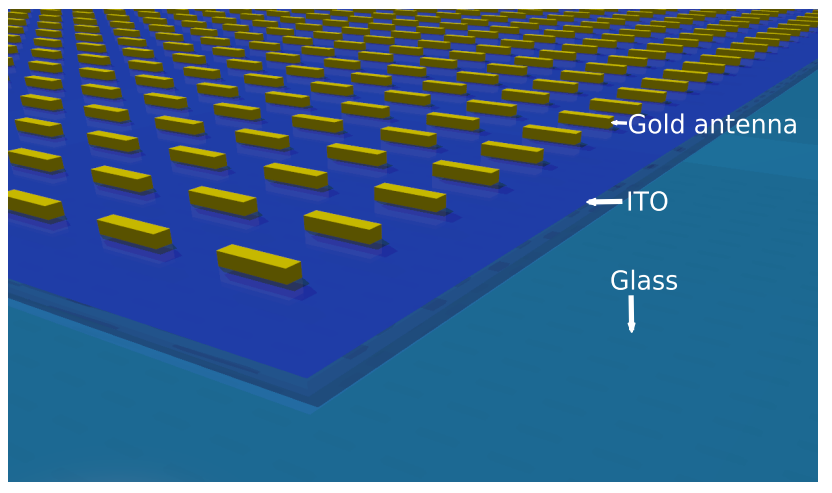

(c)

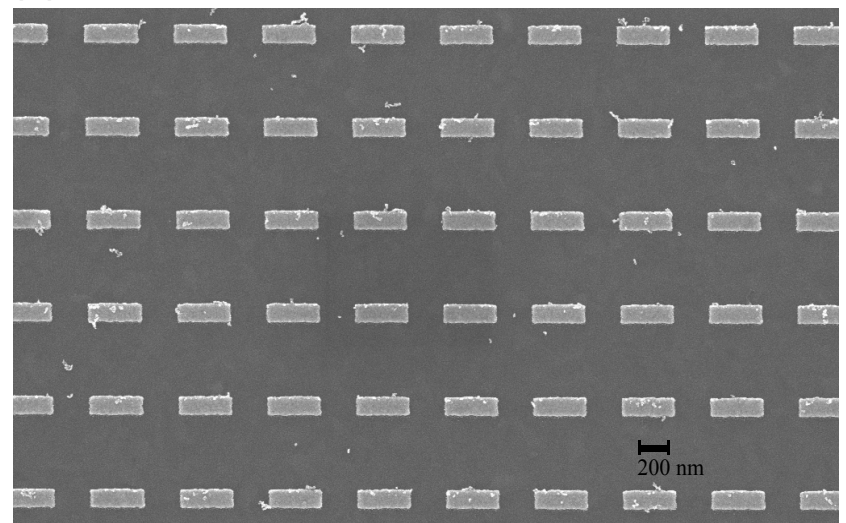

(d)

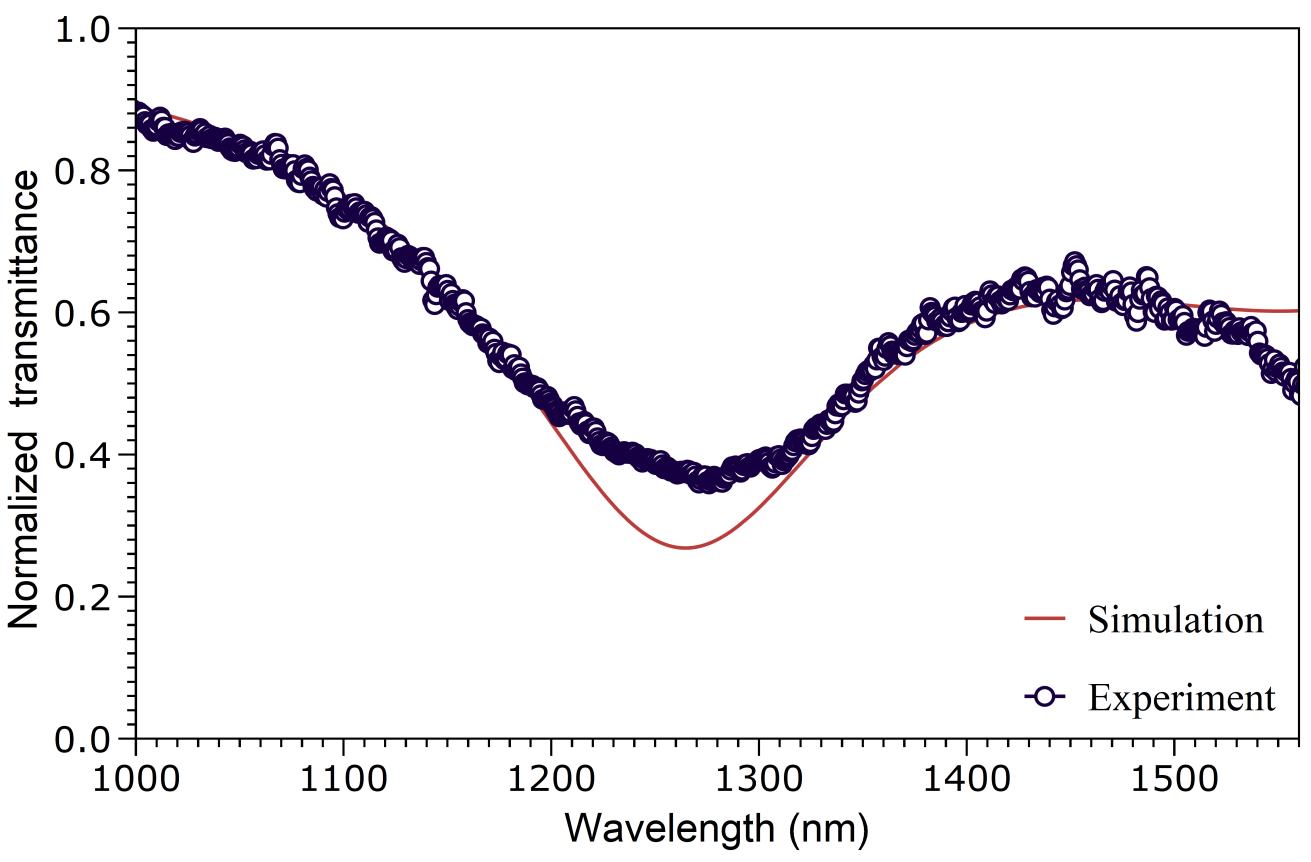



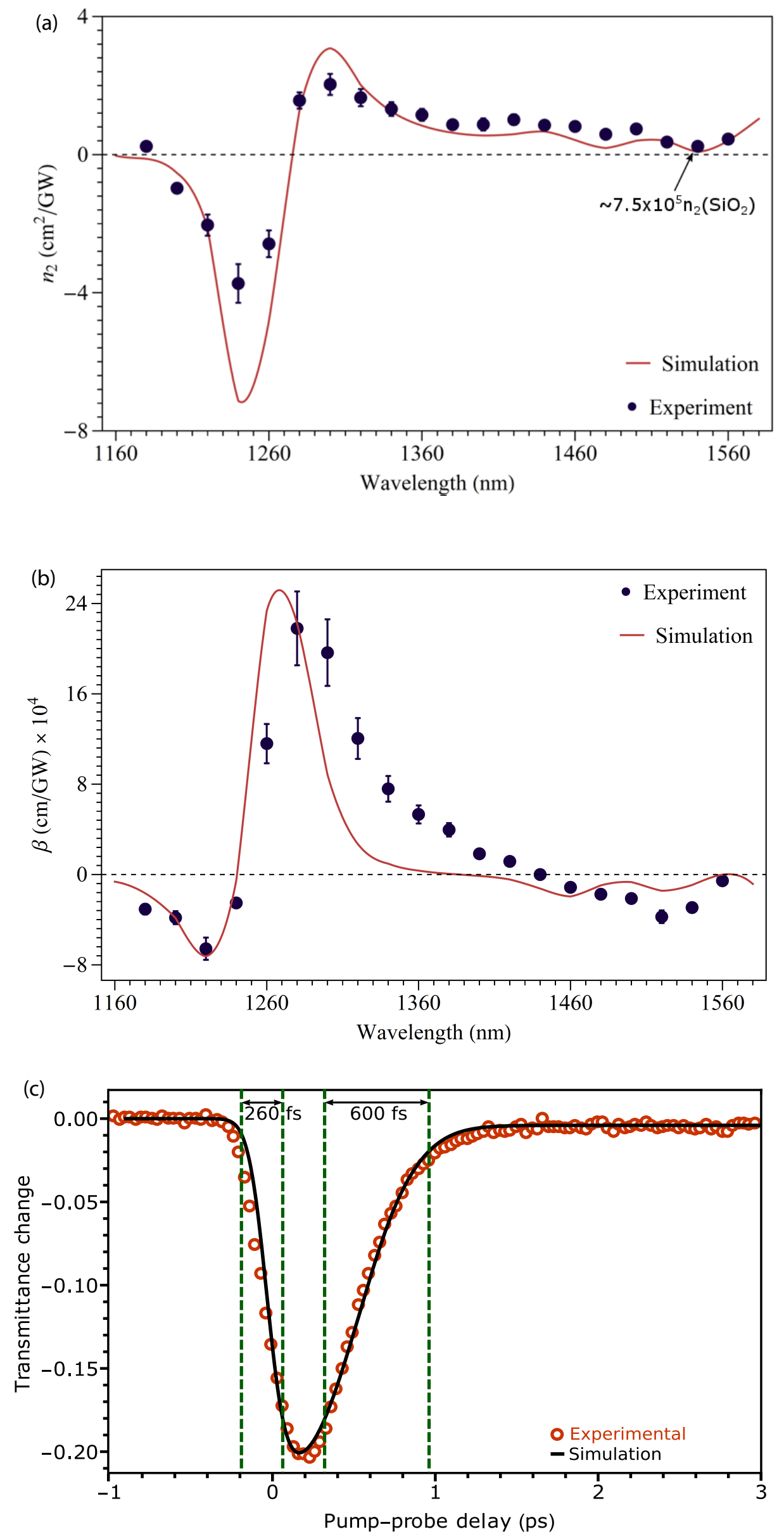


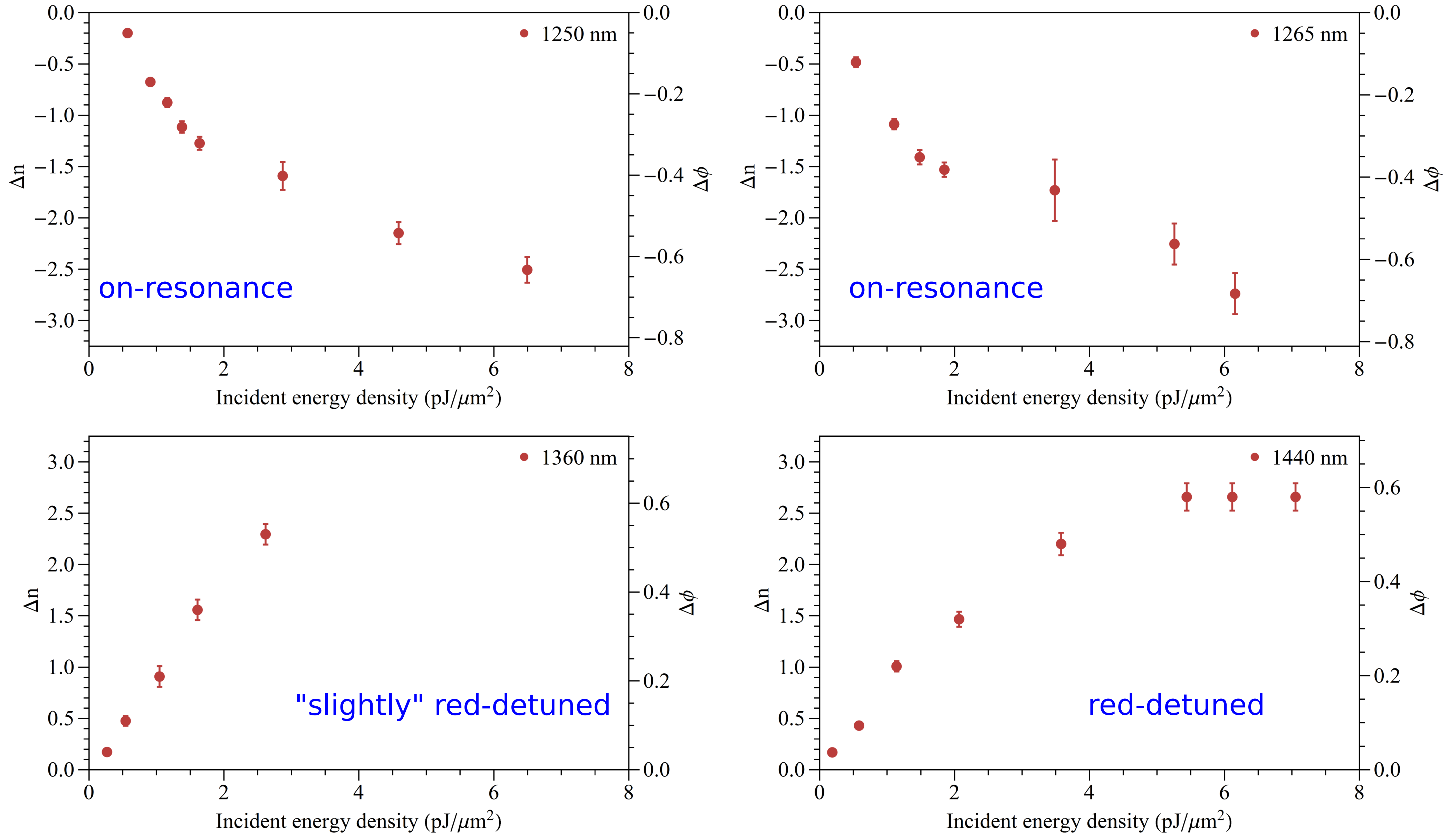

